\title{
Stigma and discrimination toward psychiatric patients
}

\section{Mini review}

According to the statistics published by WHO, 45 million suffer some sort of psychological disorders in the world. Prevalence of these disorders in general population is $25 \%$ in one' life. ${ }^{1}$ Psychiatric patients not only suffer from mental illness, but also from its stigma. Guffman $^{2}$ considers stigma as a deeply discrediting characteristic which reduces the bearer from a normal individual to an unstable and corrupt person. ${ }^{3}$ In a small qualitative study about one-third reported that antipsychotic medication was linked to stigma and self-esteem. ${ }^{4}$ Stigma is a world-shaking matter in psychiatry owing to the fact that about 300 million clients with mental health problem do not take action as they are afraid of being stigmatized and labeled. The definition of stigma has been expanded from an individualistic focus to incorporate a set of social processes that link its components under one umbrella concept. ${ }^{5}$ Stigma is thus defined as occurring when human differences are labeled, leading to stereotyping and cognitively separating 'us' from 'them'. It also causes loss of status and discrimination resulting in reduced life opportunities within the context of a power situation that allows these processes to unfold. The spectrum of emotional reactions that the stigmatizer (e.g. disgust) and the stigmatized (e.g. shame) experience has also been added as a key component to this process. ${ }^{6}$

The term stigma overarches problems of knowledge (ignorance), attitudes (prejudice) and behavior (discrimination). Stigma reduces the rate of help-seeking and contributes to diminished access to care, deficient treatment, social exclusion and financial hardship. Additionally, psychiatric medication is commonly stigmatized and often considered ineffective by the general public. ${ }^{7}$ mental health related stigma internationally a central issue for people with mental health illness. It results in disadvantages for these individuals, in particular because mental illnesses are generally more stigmatized than other conditions. Indeed, past studies have consistently reported that many people with mental health problems suffer from a lack of mental health literacy, the general public's misunderstanding of mental illness, stigmatizing attitudes and harmful discrimination, and associated problems, including low self-esteem, loss of social opportunities, and economic inequality. ${ }^{8}$

Stigma refers to a set of negative attitudes, beliefs, and thoughts about a specific situation including psychological disorders, which is not limited, the afflicted individual and includes the family members as well. Family stigma was named by Erving Goffman as "courtesy stigma" and by Mahta and Farin as "stigma by association". 9 at first and due to the effects and symptoms of the disease; the patient finds themselves unfitted with the expectations and goals of the society. This creates a sense of shame and hopelessness and as compensation, the patient tries to conceal it by secluding from the society, which in turn, leads to deprivation from personal, social, and citizenship rights. ${ }^{10}$ People with mental illness, such as schizophrenia, may internalize negative stereotypes about mental illness and respond by self-stigmatization. ${ }^{11}$

High levels of self-stigma are associated with low levels of hope, self-esteem, self-efficacy, and quality of life. Self-stigma
Volume 5 Issue 3 - 2018

\author{
Jamileh Mohtashami \\ Department of Psychiatric Nursing, Shahid Beheshti University \\ of Medical Sciences, Iran \\ Correspondence: Jamileh Mohtashami, Department of \\ Psychiatric Nursing, Shahid Beheshti University of Medical \\ Sciences, Iran, Tel +989|22797922, \\ Email j_mohtashami@sbmu.ac.ir
}

Received: June 19,2017 | Published: May 31, 2018

may undermine adherence to treatment recommendations and decrease help-seeking behavior. Non-adherence leads to increased hospitalization, higher health care costs and is a predictor of poor outcome related to relapse rates, progressive brain damage, suicide and overall mortality. ${ }^{7,12}$ It also may interfere with rehabilitation goals, such as pursuing employment, independent living, and having a full social life. ${ }^{12}$

Using a sociological perspective, Link and Phelan postulated that perceived stigma consists of five elements (labeling, stereotyping, separation, status loss, and discrimination). These elements unfold when a less powerful, stigmatized group encounters a more powerful, stigmatizing group. ${ }^{13}$ According to social psychology models, stigma has three components of stereotypes (cognitive), prejudice (emotional), and discrimination (behavioral). Stereotypes are the knowledge structures learnt by most people within a society and are in fact representative of the general beliefs and consensus of people toward the characteristics of a certain group of that society. When a person believes in the stereotypes of a mental illness, he/she will have negative emotional reactions toward it, which shows social prejudice in the form of attitudes and values, and causes discrimination against and isolation of the patient, which places the person with mental illness in an unfavorable social situation. ${ }^{3}$ Stigma causes the person with mental illness to face problems such as finding housing, employment, access to judicial systems, and using health services, which cause isolation, sense of shame, and decrease psychosocial functions such as low self-esteem, dissatisfaction with life, and mental health problems. Moreover, stigma has a negative impact on the recovery process, treatment, seeking treatment, acceptance of psychological counseling, and adherence to drug treatment. ${ }^{14}$ Imposed discrimination and deprivation on mental patients through social norms, health insurance, and public policies with respects to the patients (assigning a separate budget for mental patients) are undeniable. ${ }^{15}$ Patients, especially those who had improved, did not want to be seen in the company of mental health staff. ${ }^{16}$ In a qualitative study patients reported that the stigma of taking antipsychotics caused them to hide their medication from others and to take it only in privacy. Stigma has been shown to be associated with unfair treatment in several areas of life of individuals with mental illness.

Some authors believe that increase of social contact decreases the patient's fear and stigmatization. In addition, aging, lower economic/ social class, lack or prior knowledge of and experience with mental 
patients increases stigmatization. Personal specifications including education level, gender, type and term of the disease, severity of the symptoms, type of familial relationship, and whether the patient lives with the family or not are among the factors effective on stigma attached to the family. ${ }^{17}$

People with mental illness experience stigma from different sources such as society, family, colleagues, and mental healthcare practitioners. In fact, majority of the stigmas are associated due to fear in the society and wrong perception of others about the disease. These perceptions and wrong images might be rooted in lack of knowledge about the nature of the mental disease in the society. The mass media may also contribute in promoting wrong perceptions of these patients as violent person with criminal inclination. These images lead to more seclusion of the patients and their families in the society.

On the other hand, cultural specifications are closely related to the issues pertinent to psychological disease, seeking for treatment, and the associated stigma. As recommended by other studies, comparing with collectivism cultures of Asian, African, and Arab countries, individualism cultures like those of the USA, Germany, and Australia has lower tendency for stigmatization of mental disorder patients. ${ }^{18}$ Individualism and collectivism refer to one's definition of oneself and their relationship with the society. Despite collective cultures, individualist cultures, due to their cultural diversity and unbounded liberty, are more tolerant toward abnormalities. People in individualist culture are more independent and tend to keep their nose out of other's business. ${ }^{19}$

A variety of efforts to decrease mental-health related stigma have been made all over the world. At the global level, the World Psychiatric Association (WPA) and the World Health Organization (WHO) conducted a global anti-stigma campaign to develop tools to make it easier to tackle stigma and discrimination. Many types of interventions at the national and local level have also been developed to reduce the mental-health-related stigma of people with mental illness, including large-scale media campaigns, short education programs, social contact, films, and simulations. Despite the development of such approaches to address mental-health-related stigma, it remains a serious problem that adversely affects the lives of people with mental health problems in many countries. Not surprisingly, there is still a harmful mental-health-related stigma. ${ }^{8}$

Psycho-education is one of the recommended interventions to improve families' knowledge and attitude toward nature of psychological disease, treatments, and improvement of communication and problem solving skills. Therapists can provide such educations, given the available facilities and time, through private or group therapy for the families. In fact, psycho-education is an educational method, through which the participants receive information about nature of the psychological disorders such as etiology, treatments, side-effects, prior knowledge, improvement and recurrent of the disease, and the like. Consequently, families would have better perception of the psychological disorders and more desire to cooperate in treatment process and motivating the patient to accept the treatments.

\section{Acknowledgments}

None.

\section{Conflict of interest}

The author declares that there is no conflict of interest.

\section{References}

1. Yaghubi H, Karimi M, Omidi A, et al. Prevalence of Mental Disorders and Demographic Factors that Influence the Freshmen Students of Tehran City Universities of Medical Sciences. Journal of Clinical Psychology. 2014;6(2):22.

2. Goffman E. Stigma: Notes on the management of spoiled identity. Simon and Schuster. 2009.

3. Ebrahimi H, Namdar H, Vahidi M. Mental illness stigma among nurses in psychiatric wards of teaching hospitals in the north west of Iran. Iran J Nurs Midwifery Res. 2012;17(7):534-538.

4. Gonzalez-Torres MA, Oraa R, Aristegui M, et al. Stigma and discrimination towards people with schizophrenia and their family members. Soc Psychiatry Psychiatr Epidemiol. 2007;42(1):14-23.

5. Griffiths KM, Carron-Arthur B, Parsons A, et al. Effectiveness of programs for reducing the stigma associated with mental disorders. A meta-analysis of randomized controlled trials. World Psychiatry. 2014;13(2):161-175.

6. Hanzawa S, Nosaki A, Yatabe K, et al. Study of understanding the internalized stigma of schizophrenia in psychiatric nurses in Japan. Psychiatry Clin Neurosci. 2012;66(2):113-120.

7. Brain C, Sameby B, Allerby K, et al. Stigma, discrimination and medication adherence in schizophrenia: Results from the Swedish COAST study. Psychiatry Res. 2014;220(3):811-817.

8. Ando S, Yamaguchi S, Aoki Y, et al. Review of mental-healthrelated stigma in Japan. Psychiatry and Clinical Neurosciences. 2013;67(7):471-482.

9. Vaghee S, Salarhaji A, Asgharipour N, et al. Effects of Psycho education on Stigma in Family Caregivers of Patients with Schizophrenia: A Clinical Trial. Evidence Based Care Journal. 2015;5(3):63-76.

10. Nojomi M, Malakouti SK, Ghanean H, et al. Mental illness stigma in city of Tehran, 2009. Razi Journal of Medical Sciences. 2010;17(78\&79):4552 .

11. Gerlinger G, Hauser M, De Hert M, et al. Personal stigma in schizophrenia spectrum disorders: a systematic review of prevalence rates, correlates impact and interventions. World Psychiatry. 2013;12(2):155-164.

12. Staring ABP, Van der Gaag M, Van den Berge M, et al. Stigma moderates the associations of insight with depressed mood, low self-esteem, and low quality of life in patients with schizophrenia spectrum disorders. Schizophr Res. 2009;115(2-3):363-369.

13. Mittal D. Sullivan G, Chekuri L, et al. Corrigan. Empirical Studies of Self-Stigma Reduction Strategies: A Critical Review of the Literature. Psychiatr Serv. 2012;63(10).

14. Thornicroft G. Mehta N, Clement S, et al. Evidence for effective interventions to reduce mental-health-related stigma and discrimination. The Lancet. 2015;387(10023):1123-1132.

15. Tavakoli S, Kaviyani H, Sharifi V, et al. An examination of cognitive, emotional and behavioral stigmatizing people with mental illness. Cognitive Science News. 2007;8(2):31-43.

16. Karshaki H, Meshkin Yazd A, Soudmand P. Validation of the Mental Illness Stigma Scale, Ibn Sina Hospital, Mashhad. J Mazandaran Univ Med Sci. 2014;24(113):73-79.

17. Sadeghi M, Kaviyani H, Rezaie R. Comparative study of the stigma of mental illness in families of patients with depressive disorder, bipolar disorder and schizophrenia. Cognitive Science News. 2004;5(2).

18. Heydari A, Meshkinyazd A, Soodmand P. Mental illness stigma: A concept analysis. Modern Care. Modern care Journal. 2014;11(3):218228.

19. Corrigan Pw, Scott B, Morris, et al. Challenging the Public Stigma of Mental Illness: A Meta-Analysis of Outcome Studies. Psychiatr Serv. 2012;63(10). 